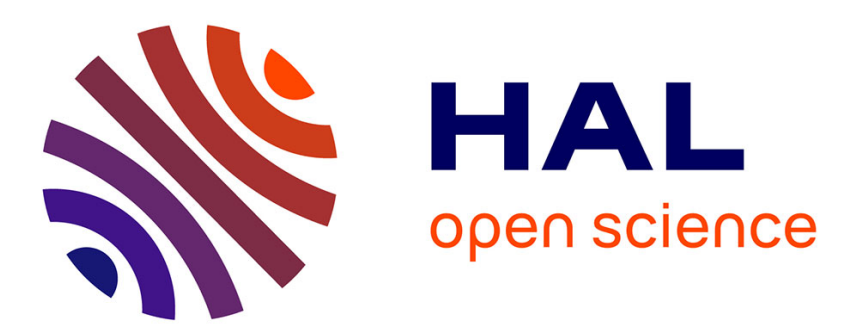

\title{
Circulating Normal IgG as Stimulator of Regulatory T Cells: Lessons from Intravenous Immunoglobulin
}

\author{
Mohan S Maddur, Srini Kaveri, Jagadeesh Bayry
}

\section{To cite this version:}

Mohan S Maddur, Srini Kaveri, Jagadeesh Bayry. Circulating Normal IgG as Stimulator of Regulatory T Cells: Lessons from Intravenous Immunoglobulin. Trends in Immunology, 2017, 38 (11), pp.789-792. 10.1016/j.it.2017.08.008 . hal-01630293

\section{HAL Id: hal-01630293 \\ https://hal.sorbonne-universite.fr/hal-01630293}

Submitted on 7 Nov 2017

HAL is a multi-disciplinary open access archive for the deposit and dissemination of scientific research documents, whether they are published or not. The documents may come from teaching and research institutions in France or abroad, or from public or private research centers.
L'archive ouverte pluridisciplinaire HAL, est destinée au dépôt et à la diffusion de documents scientifiques de niveau recherche, publiés ou non, émanant des établissements d'enseignement et de recherche français ou étrangers, des laboratoires publics ou privés. 
Circulating Normal IgG as Stimulator of Regulatory T Cells: Lessons from Intravenous Immunoglobulin

Mohan S Maddur ${ }^{1,2,3, \#, \text { Srini V Kaveri }}{ }^{1,2,3,4}$ and Jagadeesh Bayry ${ }^{1,2,3,4}$

${ }^{1}$ Institut National de la Santé et de la Recherche Médicale Unité 1138, Paris, F-75006, France

${ }^{2}$ Centre de Recherche des Cordeliers, Equipe-Immunopathologie et Immunointervention Thérapeutique, Paris, F-75006, France

${ }^{3}$ Sorbonne Universités, UPMC Univ Paris 06, UMR S 1138, Paris, F-75006, France

${ }^{4}$ Université Paris Descartes, Sorbonne Paris Cité, UMR S 1138, Paris, F-75006, France

Corresponding author: Jagadeesh Bayry (jagadeesh.bayry@ crc.jussieu.fr)

Foot note: \#Present Address: Pfizer Inc, Pearl River, NY, USA.

Keywords: Intravenous immunoglobulin; regulatory $\mathrm{T}$ cells; antibody; prostaglandin E2; dendritic cells; IL-33; Tregitopes; DC-SIGN; DCIR 


\begin{abstract}
Intravenous immunoglobulin (IVIG), a pooled normal IgG formulation prepared from the thousands of healthy donors' plasma, is extensively used for the immunotherapy of autoimmune and inflammatory disorders. Recent reports demonstrate that IVIG exerts anti-inflammatory actions by stimulating the activation and expansion of regulatory $T$ (Treg) cells by multiple mechanisms via antigen presenting cells (APCs).
\end{abstract}

$\mathrm{CD} 4{ }^{+} \mathrm{CD} 25^{+}$Foxp3+ regulatory $\mathrm{T}$ cells (Tregs) have a pivotal function in maintaining immune tolerance and lessening the inflammation-induced tissue damage. Tregs can be generated in the thymus (tTregs cells or natural Tregs) or derived in the periphery (pTregs, previously known as induced Tregs) from naive $\mathrm{CD}^{+} \mathrm{T}$ cells. As impaired generation and function of Tregs leads to severe autoimmune and/or inflammatory diseases, various approaches that improve Treg number and function are being explored to treat autoimmune diseases.

Regulation of autoimmune and inflammatory responses by circulating normal IgG: knowledge gained from IVIG

Immunoglobulin $\mathrm{G}(\mathrm{IgG})$ is a predominant class of antibodies in the circulation and one of the major players of defense against invading pathogens. Accordingly, deficiency of $\operatorname{IgG}$, as in the case of common variable immunodeficiency (CVID) or X-linked agammaglobulinemia, leads to increased predisposition to recurrent infections. Conversely, immunodeficiencies are also associated with autoimmunity and inflammatory conditions, 
suggestive of a dysregulated immune status. Intriguingly, replacement therapy with low-dose intravenous immunoglobulin (IVIG, $400 \mathrm{mg} / \mathrm{kg}$ ) consisting of intact IgG molecules purified from the pooled plasma of several thousand healthy blood donors, not only prevents recurrence of infectious diseases but also suppresses autoimmune and inflammatory responses. These observations underline the crucial regulatory role of circulating normal IgG in the maintenance of immune homeostasis.

Although initially conceived for the IgG replacement therapy of primary and secondary immunodeficiencies, high-dose IVIG $(1-2 \mathrm{~g} / \mathrm{kg})$ is now used for the immunotherapy of many autoimmune and inflammatory diseases including Guillain-Barré syndrome (GBS), Kawasaki disease, myositis, immune thrombocytopenic purpura, chronic inflammatory demyelinating polyradiculoneuropathy and others [1]. Several non-exclusive mechanisms have been proposed to explain the therapeutic effects of IVIG in these diseases, including the inhibition of activation of innate immune cells like dendritic cells (DC), macrophages and monocytes, the suppression of effector T cells such as Th1 and Th17, and the inhibition of inflammatory cytokines, complement pathway and autoantibody production.

\section{IVIG targets Tregs to exercise anti-inflammatory actions in autoimmune diseases}

Several recent reports demonstrate that high-dose IVIG therapy also stimulates the activation and expansion of Tregs, and enhances their functions to inhibit autoimmune and inflammatory responses $[2,3]$. This action of IVIG on Tregs correlates with its therapeutic benefits both in autoimmune disease patients and experimental models [2, 4]. High-dose IVIG therapy exerts sustained effect on Tregs and despite a gradual decline; this effect goes beyond the half-life of infused IgG in majority of the patients who respond to this therapy [4]. As IVIG is nothing but pooled IgG from normal donors, the effect of IVIG on Tregs likely represents a primordial function of circulating $\mathrm{IgG}$ in regulating immune homeostasis. 


\section{Treg expansion by IVIG: How many mechanisms after all?}

Dissection of underlying mechanisms by which high-dose IVIG expands Tregs has led to identification of multiple mechanisms (Figure 1).

Cyclooxygenase (COX)-2-dependent-Treg expansion by IVIG: Our in vitro data demonstrate that by signaling through DC-SIGN, IVIG and its $\left.\mathrm{F}(\mathrm{ab})_{2}\right)_{2}$ fragments at concentrations corresponding to amount of IgG received by the patients under high-dose IVIG therapy, expand human Tregs by inducing COX-2-dependent prostaglandin E2 ( $\left.\mathrm{PGE}_{2}\right)$ secretion in DC [5]. Furthermore, Treg expansion and disease ameliorative ability of IVIG in vivo were compromised in experimental autoimmune encephalomyelitis (EAE) mouse model upon COX-2 inhibition. Investigations in autoimmune patients also confirmed the induction of $\mathrm{PGE}_{2}$ following high-dose IVIG therapy [6].

Dendritic cell immunoreceptor (DCIR)-dependent induction of Tregs: In a mouse model of airway hyperresponsiveness, IVIG at $2 \mathrm{~g} / \mathrm{kg}$ dose significantly enhanced pTregs in vivo in the lungs [7]. Mechanistically, pTreg induction by IVIG required interaction of $\alpha(2,6)$-sialic acid linkages of $\operatorname{IgG}$ (either at Fc or Fab region) with DCIR on CD11c ${ }^{+}$DC. SIGN-R1 (murine ortholog of DC-SIGN) on the other hand was dispensable. Interference with DCIR-binding or DCIR-signaling abrogated IVIG-mediated Treg induction by $\mathrm{CD} 11 \mathrm{c}^{+} \mathrm{DC}$ upon adoptive transfer and attenuation of airway inflammation.

IL-33-dependent induction of Tregs by IVIG: A more recent study in EAE reports IL-33dependent activation and expansion of pTregs by high-dose IVIG (1 g/kg) that inhibit 
pathogenic $\mathrm{CD}^{+} \mathrm{T}$ cell responses in vivo [8]. This model further suggests that IVIG induces IL-33 in SIGN-R1 ${ }^{+}$marginal zone macrophages via interaction of $\alpha(2,6)$-sialic acid linkages of Fc fragment with SIGN-R1 (Box 1). During cognate interaction between APC (like DC) and Tregs, IL-33 in the microenvironment activates Tregs by signaling through ST2 receptor. Although IVIG therapy in autoimmune patients induces IL-33, as discussed later, the proposed model of IL-33 induction was not reproduced in human (Box 1).

Activation of Tregs by processing and presentation of IgG-derived 'Tregitopes': Data from bioinformatics, in vitro stimulation of human peripheral blood mononuclear cells and house dust mite allergy model in HLA-DR4 transgenic mice suggest the existence of highly promiscuous MHC class II T-cell epitopes in the Fc and Fab regions of IgG (and hence in IVIG) with regulatory features called "Tregitopes" [9]. These Tregitopes are processed and presented by APC to activate tTregs. It is not yet clear however whether such mechanism is directly implicated in the amelioration of autoimmune response and Treg stimulation in IVIG-treated patients.

Thus, irrespective of aforementioned mechanisms, APC such as DC and C-type lectin receptors on them appear to be chief architects of IVIG-mediated Treg responses both in humans and animal models. While human data advocate mechanisms for tTreg expansion, murine data provide insight on pTregs. Implication of both tTregs and pTregs in the inhibition of autoimmune response and inflammation, favors the existence of multiple mechanisms of Treg induction by IVIG. Findings in human further echo this possibility as IVIG therapy in a small cohort of GBS patients significantly induced both PGE $_{2}$ and IL-33 [6]. It is also important to note that IVIG could directly stimulate human Treg expansion in vitro under the influence of co-stimulatory signals. 
Mouse data suggest that at least two pathways of pTreg induction are under operation: a direct pathway involving the interaction of sialylated IgG with DCIR on DC and an indirect pathway dependent on IL-33 via interaction of sialylated Fc with SIGN-R1 on marginal zone macrophages [7, 8]. As SIGN-R1 is not expressed by mouse DC, DCIR seems to be the major receptor for IVIG on murine DC. The mechanism(s) by which DCIR ${ }^{+}$DC induce Tregs is open for further exploration.

However, unlike in mouse, in vitro differentiated human DC express both DC-SIGN and DCIR. Therefore, which receptor has a dominant role remains unclear, although current data favors a dominant role of DC-SIGN in mediating Treg expansion via COX-2-PGE 2 pathway [5]. We propose that either DCIR has a redundant role in human Treg expansion in the context of inflammatory DC or that the relative contribution of these two lectin receptors is determined by subsets of DC and tissues, as it is important to note here that DC ex vivo express mainly DCIR.

\section{Why does Treg expansion by IVIG mimic the function of circulating IgG?}

IVIG symbolizes a complete repertoire of normal circulating IgG. The distribution of IgG subclasses and IgG glycosylation patterns in IVIG generally overlaps with normal human plasma/serum. Whereas a single donor might lack certain individual IgG specificities, that is likely to be compensated in IVIG because of pooling of plasma.

The immunodeficient patients either lack or have reduced amount of $\mathrm{IgG}$ in the circulation. As a corollary, such conditions are also associated with reduced frequency of Tregs and/or Foxp3 expression [10]. Of note, replacement therapy with IVIG (400 mg/kg) aiming at bringing up the IgG level in immunodeficient patients close to that of healthy individuals, transiently (and temporarily) enhances Tregs immediately after therapy [11]. These results 
point out that circulating IgG are indeed critical for maintaining Tregs. But to achieve this, it is important to maintain normal levels of $\operatorname{IgG}$ constantly. This paradigm might explain why long-term repetitive IVIG replacement therapy in immunodeficient patients did not reveal consistent effect on Tregs [12].

The most debated question is, if IgG are capable of inducing Tregs, then despite having them in the blood at normal levels, why do autoimmune patients require high-dose IVIG therapy to ameliorate autoimmune condition and to expand Tregs?

It appears that the high-dose requirement for IVIG to treat autoimmunity rests in the composition of IVIG itself. In autoimmunity and inflammation, both innate and adaptive immune cells are hyper-activated, while regulatory mechanisms are either dysfunctional or reduced in their ability to control these pathogenic responses. Natural antibodies and natural autoantibodies with low to medium affinity are likely to be the major active components of IVIG, but these specificities are not in high frequencies. Thus, given the altered physiology in autoimmune patients, it is conceivable that these natural autoantibodies are needed at higher amounts than those present in the normal circulation of a donor. Moreover, IgG also lose sialic acid content during affinity maturation and inflammation. These requirements likely underlie the requirement for high (1-2 g/kg) doses of IVIG to treat autoimmune conditions, while in healthy individuals, physiological levels of $\operatorname{IgG}$ with a normal frequency of natural autoantibodies and sialylation, are sufficient to maintain homeostasis.

\section{Conclusion}

In summary, emerging evidences have revealed that multiple pathways are targeted by IVIG (or circulating normal $\mathrm{IgG}$ ) to propagate Treg expansion. Further work is necessary to translate the experimental findings into the clinic and to harness the pathways of Treg 
expansion as potential biomarkers of IVIG response, as, other than $\mathrm{PGE}_{2}$ and IL-33, the other mechanisms are not yet investigated in the patients.

\section{Acknowledgments}

Supported by Institut National de la Santé et de la Recherche Médicale (INSERM), Centre National de la Recherche Scientifique (CNRS), Université Pierre et Marie Curie and Université Paris Descartes. Because of space limitations, we could only cite key reports and apologize to colleague whose important work could not be discussed here.

Conflict of interests: Dr Bayry and Dr Kaveri received research funding from CSL Behring (Switzerland and France) and Laboratoire Français du Fractionnement et des Biotechnologies, France. 


\section{References}

1. Gelfand, EW. (2012). Intravenous immune globulin in autoimmune and inflammatory diseases. N. Engl. J. Med. 367:2015-2025.

2. Ephrem, E. et al. (2008). Expansion of CD4+CD25+ regulatory T cells by intravenous immunoglobulin: a critical factor in controlling experimental autoimmune encephalomyelitis. Blood 111:715-722.

3. Tjon, AS. et al. (2013). Patients treated with high-dose intravenous immunoglobulin show selective activation of regulatory T cells. Clin. Exp. Immunol. 173:259-267.

4. Hirabayashi ,Y. et al. (2013). Lack of $\mathrm{CD} 4^{+} \mathrm{CD} 25^{+} \mathrm{FOXP} 3^{+}$regulatory $\mathrm{T}$ cells is associated with resistance to intravenous immunoglobulin therapy in patients with Kawasaki disease. Eur. J. Pediatr. 172:833-837.

5. Trinath, J. et al. (2013). Intravenous immunoglobulin expands regulatory $\mathrm{T}$ cells via induction of cyclooxygenase-2-dependent prostaglandin E2 in human dendritic cells. Blood 122:1419-1427.

6. Maddur, M.S. et al. (2017). Regulatory T cell frequency, but not plasma IL-33 levels represents potential immunological biomarker to predict clinical response to intravenous immunoglobulin therapy. J. Neuroinflammation 14: Article number 58. doi: 10.1186/s12974-017-0818-5.

7. Massoud, A.H. et al. (2014). Dendritic cell immunoreceptor: a novel receptor for intravenous immunoglobulin mediates induction of regulatory T cells. J. Allergy Clin. Immunol. 133:853-863.

8. Fiebiger, B.M. et al. (2015). Protection in antibody- and T cell-mediated autoimmune diseases by antiinflammatory IgG Fcs requires type II FcRs. Proc. Natl. Acad. Sci. U S A. 112:E2385-E2394.

9. De Groot, A.S. et al. (2008). Activation of natural regulatory T cells by IgG Fc-derived peptide "Tregitopes". Blood 112:3303-3311

10. Genre, J. et al. (2009). Reduced frequency of $\mathrm{CD}^{+} \mathrm{CD} 25^{\mathrm{HIGH}} \mathrm{FOXP} 3^{+}$cells and diminished FOXP3 expression in patients with common variable immunodeficiency: a link to autoimmunity? Clin. Immunol. 132:215-221.

11. Kasztalska, K. et al. (2011). Intravenous immunoglobulin replacement therapy in the treatment of patients with common variable immunodeficiency disease: an open-label prospective study. Clin. Drug Investig. 31:299-307.

12. Paquin-Proulx, D. et al. (2013). IVIg immune reconstitution treatment alleviates the state of persistent immune activation and suppressed CD4 T cell counts in CVID. PLoS One 8:e75199.

\section{BOX 1}




\section{Is Fc-sialylation critical for IVIG-mediated anti-inflammatory actions and Treg expansion?}

Structurally, $\mathrm{IgG}$ is divided into $\mathrm{F}(\mathrm{ab})_{2}$ fragment that binds to specific antigen, and Fc region that binds to diverse Fc $\gamma$ Rs present on innate immune cells and B cells to mediate effector functions. Being glycoprotein, all IgG are glycosylated at Asn297 of Fc region (in the CH2 domain).

Mechanistically, both $\mathrm{Fc}$ and $\left.\mathrm{F}(\mathrm{ab})_{2}\right)_{2}$-mediated anti-inflammatory mechanisms of IVIG have been reported. Recently, Fc-dependent action of IVIG was proposed to be mediated mainly by a fraction of IgG that contains terminal $\alpha(2,6)$-sialic acid linkages at Asn297. These $\alpha(2,6)$-sialic acid linkages interact with C-type lectin receptors such as DC-SIGN (SIGN-R1), DCIR or Siglecs like CD22. However, $15-20 \%$ of IgG are also glycosylated at Fab region (in $\mathrm{V}_{\mathrm{H}}$ and $\mathrm{V}_{\mathrm{L}}$ regions) and few reports show that $\alpha(2,6)$-sialic acid linkages of Fab but not Fc fragments are important for anti-inflammatory effects of IVIG. Current evidences clearly indicate that Fc-sialylation-mediated action represents 'one of the' pleiotropic antiinflammatory effects of IVIG rather than being 'only' the mechanism. Several groups have reported that sialylation is not a pre-requisite and IVIG devoid of Fc-sialylation also mediate anti-inflammatory actions. This is not surprising as IVIG is not a preparation with single IgG specificity rather it is a heterogeneous $\operatorname{IgG}$ preparation and hence acts on almost all compartments of the immune system.

Is Fc-sialylation critical for IVIG-mediated Treg expansion in humans? The answer remains unclear. SIGN-R1 that is identified as one of the lectin receptors for the recognition Fc$\alpha(2,6)$-sialic acid linkages, is expressed mainly by splenic marginal zone macrophages. On the contrary, DC-SIGN in humans is expressed predominantly on DC. Distinct expression 
pattern of DC-SIGN and SIGN-R1, and differences in the splenic architecture of human and mouse are the major hurdles in translating Fc-sialylation data of mouse to human.

In vitro data from human monocyte-derived DC unequivocally support interaction of IVIG with DC-SIGN for Treg expansion. But it might be mediated via either sialylated glycans of Fab region or interaction of DC-SIGN-specific natural autoantibodies in the IVIG. Furthermore, unlike mouse, DC-SIGN ${ }^{+}$DC (either monocyte-derived or from the spleen) did not secrete IL-33 upon IVIG exposure in vitro. But analyses of autoimmune patients' plasma following high-dose IVIG therapy confirmed that IL-33 is induced. Therefore, it is likely that endothelial or epithelial cells but not $\mathrm{DC}-S I G N^{+}$cells might have contributed to IL-33 increase seen in the patients. Taken together, Fc-sialylation might have a role in Treg expansion via IL-33 induction, but the proposed pathway is not true in humans. 


\section{Figure 1. Proposed mechanisms of induction and expansion of Tregs by IVIG}

Intravenous immunoglobulin (IVIG) is a pooled normal IgG preparation. Several reports have now provided evidence that interaction of $\operatorname{IgG}$ molecules with antigen presenting cells (APC) such dendritic cells (DC) and macrophages via C-type lectin receptors (CLRs), constitutes first step of regulatory T cell (Treg) induction and expansion by IVIG. Internalized IVIG then targets multiple pathways to modulate the phenotype and function of APC and to induce and expand Tregs. Thus, underlying mechanisms by which high-dose IVIG expands Tregs diverge depending on the experimental models used: autoimmune vs inflammatory conditions; and model species, i.e., human vs mouse. (a) Interaction of IVIG with DC-SIGN through $\mathrm{F}(\mathrm{ab})_{2}$ fragments (but not $\mathrm{Fc}$ fragments) signals cyclooxygenase (COX)-2dependent prostaglandin $\mathrm{E} 2\left(\mathrm{PGE}_{2}\right)$ secretion in $\mathrm{DC}$ to expand tTregs. Inhibition of $\mathrm{PGE}_{2}$ synthesis by COX-2 inhibitors or blocking IVIG interaction with DC-SIGN prevented IVIGtriggered Treg expansion by human DC. (b) Cross-talk between $\alpha(2,6)$-sialic acid linkages of IgG (either at $\mathrm{Fc}$ or Fab region) with DCIR on murine $\mathrm{CD} 11 \mathrm{c}^{+} \mathrm{DC}$ initiates downstream signaling through Src homology domain 2-containing protein tyrosine phosphatase 2 (SHP-2) to induce tolerogenic status of DC and de novo generation of pTregs with enhanced suppressive function. (c) IVIG induces IL-33 in SIGN-R1 ${ }^{+}$innate cells via interaction of $\alpha(2,6)$-sialic acid linkages at Asn297 of Fc fragment with SIGN-R1, and IL-33 in turn mediates the de novo generation of pTregs in EAE model. (d) Activation and expansion of tTregs by Tregitopes present on IgG molecules. APC such as DC endocytose IgG (probably via C-type lectin receptors), process IgG in the intracellular MHC-II compartment and present Tregitopes in the context of MHC class II that specifically activate Tregs. In addition to activation and expansion of Tregs, IVIG also modulates APC and can act at the T-cell level to interfere with polarization and function of pathogenic Th cell subsets (Th1, Th2 and Th17 cells) to exert therapeutic beneficial effects. 


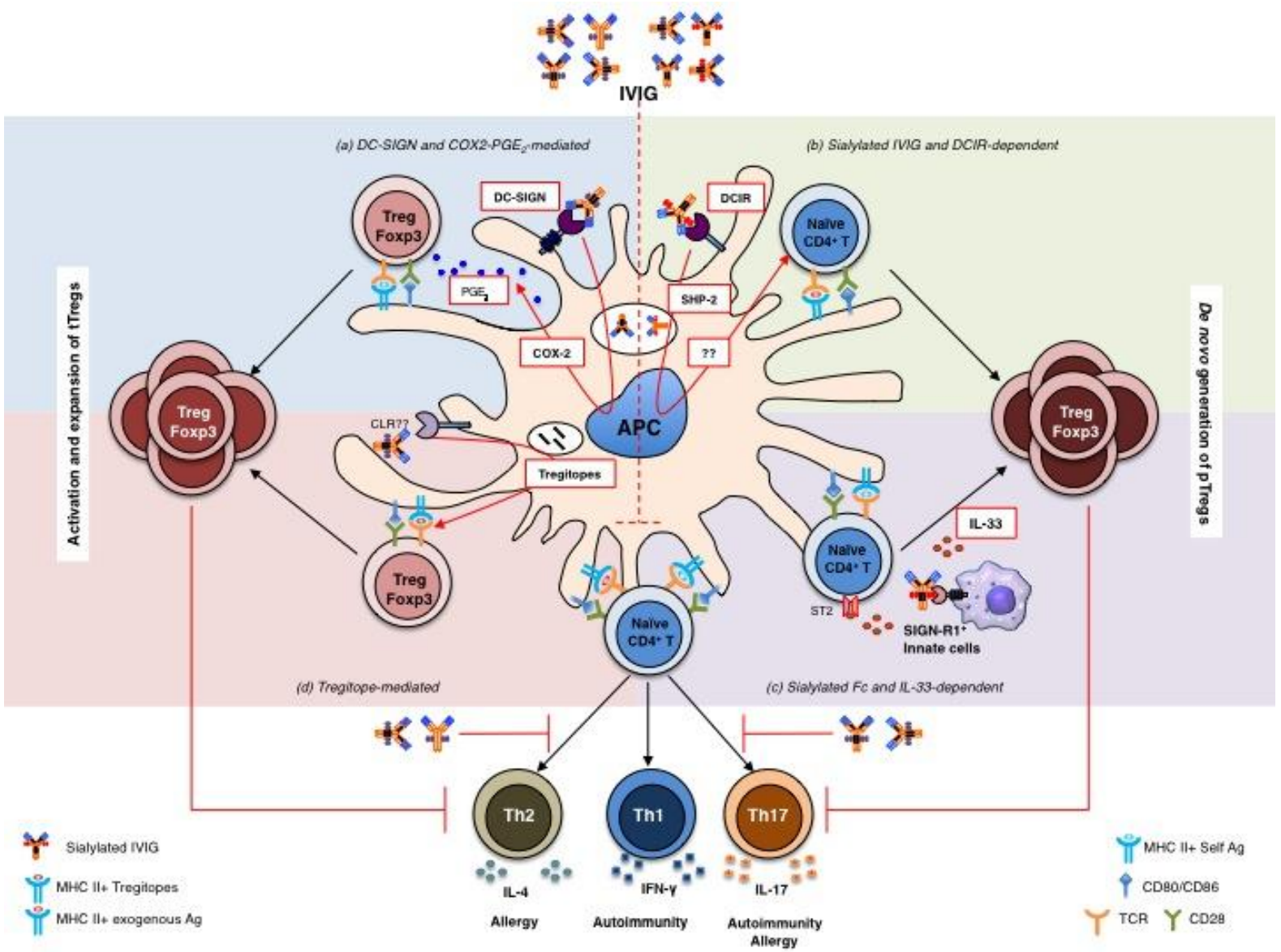

